\title{
Non-coding RNAs regulate tumor cell plasticity
}

\author{
LIU BoDu, SUN LiJuan \& SONG ErWei* \\ Breast Tumor Center, Sun Yat-sen Memorial Hospital, Sun Yat-sen University, Guangzhou 510120, China
}

Received August 1, 2013; accepted September 10, 2013

\begin{abstract}
Tumor metastasis is one of the most serious challenges for human cancers as the majority of deaths caused by cancer are associated with metastasis, rather than the primary tumor. Recent studies have demonstrated that tumor cell plasticity plays a critical role in tumor metastasis by giving rise to various cell types which is necessary for tumor to invade adjacent tissues and form distant metastasis. These include differentiation of cancer stem cells (CSCs), or epithelial-mesenchymal transition (EMT) and its reverse process, mesenchymal-epithelial transition (MET). A growing body of evidence has demonstrated that the biology of tumor cell plasticity is tightly linked to functions of non-coding RNAs (ncRNAs), especially microRNAs (miRNAs) and long non-coding RNAs (lncRNAs). Therefore, understanding the mechanisms how non-coding RNAs regulate tumor cell plasticity is essential for discovery of new diagnostic markers and therapeutic targets to overcome metastasis.
\end{abstract}

metastasis, tumor cell plasticity, CSCs, EMT, miRNA, IncRNA

Citation: $\quad$ Liu B D, Sun L J, Song E W. Non-coding RNAs regulate tumor cell plasticity. Sci China Life Sci, 2013, 56: 886-890, doi: $10.1007 / \mathrm{s} 11427-013-4554-5$

Tumor cell plasticity describes the ability of tumor cells to transform into other cell types. This process generates cells with new phenotypes ensuring their proliferation and metastasis. Cancer stem cells (CSCs) are cancer cells that possess characteristics associated with normal stem cells, especially the ability to give rise to all cell types found in a particular tumor [1,2].

Epithelial-mesenchymal transition (EMT) is the process by which epithelial cells lose epithelial characteristics and gain mesenchymal characteristics. During EMT, epithelial cells lose cell-cell junctions, thus facilitating cell individualization. In addition, the epithelial apical-basal polarity is lost, and a complete reorganization of the actin cytoskeleton enhances cell locomotion along rear-to-front polarity. EMT also enables cells to acquire invasive properties, thus degrading extracellular matrix and re-synthesizing extracellular matrix proteins $[3,4]$. Transcription factors Snail (Snail/
Slug), ZEB (ZEB1/ZEB2), and Twist1 act to repress Ecadherin expression, and induce mesenchymal gene expression, such as vimentin and fibronectin in this transition [5]. In carcinomas, cancer cells can undergo EMT to escape the primary tumor, invade surrounding tissues, and eventually colonize remote sites via blood or lymphatic routes to generate metastases. Metastatic cells can then revert through mesenchymal-epithelial transition (MET) to re-acquire epithelial characteristics similar to cells in the primary tumor.

Therefore, development of specific therapies targeted at CSCs and EMT holds hope for improvement of survival and quality of life of cancer patients, especially for sufferers of metastatic disease. The molecular and cellular mechanisms underlying CSCs and EMT are complex. The discovery that non-coding RNAs (ncRNAs) are powerful regulators of CSCs and EMT has added an important new layer to our understanding of tumor cell plasticity and the regulation of these processes in cancer.

*Corresponding author (email: songew@mail.sysu.edu.cn) 


\section{Classification of ncRNAs}

Non-coding RNAs (ncRNAs) are RNA molecules that do not function by encoding for proteins. They are loosely grouped into two major classes based on their size: small ncRNAs less than $200 \mathrm{nt}$, and long ncRNAs (IncRNAs) longer than 200 nucleotides (nt) [6,7]. Small ncRNAs are represented by a broad range of known and newly discovered RNA species [8,9]. These include well-characterized housekeeping ncRNAs such as tRNA and rRNA which are essential for fundamental cellular functions, splicing RNAs (snRNAs) that regulates mRNA splicing, and a variety of recently-observed RNAs regulating transcription, such as tiny transcription-initiation RNAs, promoter-associated short RNAs and termini-associated short RNAs. However, the most extensively studied small RNAs in cancer are microRNAs (miRNAs) [6,7].

miRNAs are small double-strand RNAs at 22 nt long. They regulate protein expression at post-transcriptional level by binding to the 3'UTRs of target mRNAs, which leads to translation repression or target mRNA cleavage [10,11]. miRNAs are grouped into families based on similarity of seed sequences. Members of a family are expected to have many overlapping targets. Therefore, individual miRNAs often target multiple mRNAs within a common pathway, increasing the robustness of their effects on cellular processes such as EMT, while single mRNAs can be regulated by multiple miRNAs [12].

In contrast to miRNAs, IncRNAs are mRNA-like transcripts ranging in length from $200 \mathrm{nt}$ to $\sim 100$ kilobases $(\mathrm{kb})$ lacking significant open reading frames. Many identified lncRNAs are transcribed by RNA polymerase II (RNA pol II) and are polyadenylated [13,14]. Transcription of lncRNAs occurs from an independent gene promoter and is not coupled to the transcription of a nearby or associated parental gene, as with promoter/termini associated RNAs [6]. Although only a minority have been characterized in detail, lncRNAs participate in diverse biological processes through distinct mechanisms. Generally, IncRNAs have been implicated in chromosome dosage-compensation, imprinting, epigenetic regulation, cell cycle control, nuclear and cytoplasmic trafficking, transcription, translation, splicing, cell differentiation, and others [15-19].

\section{2 miRNA regulation of CSCs and EMT}

Breast CSCs (BCSCs) were the first CSCs in solid tumors to be reported and are among the best characterized CSCs [20]. BCSCs can be enriched by consecutively passaging breast cancer cell SKBR3 in mice treated with chemotherapy [21]. These xenografts contain a high percentage of stem-like $\mathrm{CD} 44^{+} / \mathrm{CD} 24^{-}$cells. Importantly, the BCSCenriched cells expressed much lower levels of let-7 as well as a number of other miRNAs, including miR-16, miR-107, miR-128, and miR-20b, than the parental cells. let-7 regulated self-renewal and differentiation of BCSCs by targeting H-RAS and HMGA2, respectively. Overexpression of let-7a inhibited mammosphere formation, tumor formation, and metastasis in NOD/SCID mice and reduced the proportion of undifferentiated cells in vitro. Subsequently, miR-30 was also found to be markedly reduced in BCSCs and to negatively modulate the stemness of BCSCs. Overexpression of miR-30 in BCSCs not only diminished their self-renewal ability but also reduced anoikis resistance and increased apoptosis by targeting UBC9 and integrin $\beta 3$. A more complete inhibition of self-renewal in BCSCs was observed when both let-7 and miR-30 were introduced at the same time, compared with transfecting either miRNA alone. The synergistic inhibitory effects of let-7 and miR-30 suggest that multiple miRNAs may distinctively and concertedly regulate CSC properties [22].

Furthermore, miR-17-19b is up-regulated in leukemia stem cells, consistent with reduced differentiation and increased proliferation, in part by modulating the expression of p21 [23]. miR-34a was found to be down-regulated in human glioblastomas. Transfection of miR-34a into glioblastoma multiforme (GBM) CSCs caused cell-cycle arrest or apoptosis and also inhibited xenograft growth, mediated by down-regulation of multiple oncogenic targets, including c-MET, Notch-1/2, and CDK6 [24]. The miR-181 is important for the maintenance of hepatic cancer stem cells by down-regulating two hepatic transcriptional regulators promoting differentiation (CDX2 and GATA6) and an inhibitor of Wnt/ $\beta$-catenin signaling (NLK) [25].

Several miRNAs directly target families of EMT transcription factors. For example, the transcription factors ZEB1 and ZEB2 have been confirmed as targets of miR-200 family [26,27]. Conversely, ZEB1 represses the transcription of miR-200 genes by binding to their promoter region, thereby forming a double-negative feedback loop [28]. A striking negative correlation was found between ZEB and miR-200 expression in many human cancer cell lines. miR-205 was also reported to inhibit ZEB1 and ZEB2 expression [26]. Snail is targeted by miR-29b and miR-30a $[29,30]$. Accordingly, enhanced expression of miR-29b in metastatic prostate cancer cells reverses EMT and inhibits the invasive phenotype. Furthermore, miR-1 and miR-200b both directly target Slug in prostate adenocarcinoma cells [31]. In this system, TGF- $\beta$-induced EMT results in expression of Slug, which in turn transcriptionally represses the expression of both miR-1 and miR-200b, forming a double negative feedback loop. miR-200b and miR-15b were down-regulated in cisplatin-resistant tongue squamous cell carcinoma (TSCC) cells, which show the acquisition of EMT phenotype. Re-expression of miR-200b and miR-15b induced MET with up-regulated E-cadherin expression, down-regulated vimentin and fibronectin expressions, and inhibited invasion and migration in cisplatin-resistant cells 
by targeting BMI1. In vivo, miR-200b or miR-15b suppressed metastasis of TSCC xenografts established by cisplatin-resistant cells [32].

Mani et al. [33] first firmly connected EMT and CSCs by inducing HMLE cells to undergo EMT by Snail or Twist and revealed that the EMT markers are highly expressed in human mammary $\mathrm{CSCs}$ that were $\mathrm{CD} 44^{\text {high }} / \mathrm{CD} 24^{\text {low }}$ and were able to form mammospheres. Subsequently, the connection of EMT and CSCs was demonstrated in many other human cancers. miR-21 acts as oncogenic miRNA, and its expression promotes EMT and CSC characteristics in breast cancer cells, notably through inhibition of PTEN expression $[34,35]$. Expression of the miR-106b-25 cluster, which acts downstream of the transcription factor Six1, induces EMT and tumor-initiating characteristics in human breast cancer cells, through direct repression of Smad7, thus increasing TGF- $\beta$ signaling [36]. Linking EMT and CSCs to specific miRNAs will enable us to better understand how metastatic cancer arisen and targeting these regulatory miRNAs may provide new ways to overcome cancer.

\section{IncRNAs in cancer}

According to recent results from ENCODE project, $62 \%$ of human genome is coding for transcripts longer than $200 \mathrm{nt}$, yet only $5.5 \%$ of them is represented by coding exons. It is estimated that the total number of IncRNAs is between 10000 and 200000 [37]. However, only very few examples of IncRNAs have been functionally characterized [15,38]. Recent studies show that lncRNAs are exquisitely regulated during development and in response to diverse signaling cues, and can be misexpressed in solid tumors and leukemias [39], suggesting that lncRNAs constitute an important component of tumor biology. One of the first lncRNAs described to have fundamental roles in cancer was the HOX antisense intergenic RNA (HOTAIR), encoded by a $2.2 \mathrm{~kb}$ gene located in the mammalian HOXC locus on chromosome 12q13.13 [40]. This IncRNA was found to be highly up-regulated in both primary and metastatic breast tumors. High levels of HOTAIR expression were correlated with both metastasis and poor survival rate [41].

The MALAT1 gene, or metastasis-associated lung adenocarcinoma transcript 1 , was first associated with high metastatic potential and poor patient prognosis during a comparative screen of non-small cell lung cancer patients with and without metastatic tumors [42]. Recently, Ying et al. [43] found that MALAT-1 levels were up-regulated in bladder cancer tissues compared with adjacent normal tissues, and siRNA-mediated MALAT-1 silencing resulted in a decrease of ZEB1, ZEB2 and Slug levels, and an increase of E-cadherin levels. MALAT-1 promoted EMT by activating Wnt signaling in vitro. These data suggest a potential application of MALAT-1 in cancer therapy.

Several lncRNAs are implicated in the regulation of p53 tumor suppressor signaling. MEG3, a maternally-expressed imprinted lncRNA on Chr14q32, has been shown to activate p53 and facilitate p53 signaling, including enhancing p53 binding to target gene promoters [44]. MEG3 overexpression suppresses cell proliferation in meningioma [45] and hepatocellular carcinoma cell lines [46]. A recently described murine lncRNA located near the p21 gene, termed linc-p21, has also emerged as a promising p53-pathway gene. In murine lung, sarcoma, and lymphoma tumors, linc-p21 expression is induced upon activation of p53 signaling and represses p53 target genes through a physical interaction with hnRNP-K, a protein that binds the promoters of genes involved in p53 signaling. linc-p21 is further required for proper apoptotic induction [47]. These two lncRNAs may be a putative tumor suppressor.

Recent research has shed light on the possibilities that lncRNAs could regulate crucial signal pathways in tumor cell biology. IncRNA low expression in tumor (lncRNALET) is down-regulated in hepatocellular carcinomas and other types of cancers. It is down-regulated by hypoxia-induced histone deacetylase 3, and down-regulation of lncRNA-LET enables stabilization of nuclear factor 90 protein, which leads to hypoxia-induced cell invasion [48]. Another study shows that two lncRNAs, PRNCR1 and PCGEM1, bind to androgen receptor and are required for ligand-dependent activation of androgen receptor pathway. Moreover, their pathological up-regulation can trigger ligand-independent activation of androgen receptor pathway and may facilitate castration resistance in prostatic tumors [49]. These examples have raised the possibility that lncRNAs may play significant roles in tumor cell plasticity by being involved in regulation of critical signal pathways.

\section{Concluding remarks and future perspectives}

Malignant tumors are heterogeneous in their cellular components. Tumor cells vary in their tumorigenicity, ability to metastasis, and resistance to therapies. This nature of tumor cells is governed by both genetic heterogeneity and cellular plasticity. Growing evidence suggests that cellular plasticity is tightly linked to tumor progression, metastasis, resistance to therapies, and relapse. Recent studies show non-coding RNAs played a critical role in regulating tumor cell plasticity. These mainly include miRNAs and lncRNAs, as they exert their functions by regulating gene expression which is essential mechanism of cell plasticity (Table 1). Future explorations of expressions and functions of these expression-regulating molecules in human cancer may lead to discoveries of new markers for diagnosis or developments of targeted therapeutics.

Challenges of analyzing functions of cancer related ncRNAs mainly involve specifically modulation of their expressions. While genetic knock-outs of certain protein-coding genes are often achieved by introducing a stop 
Table 1 ncRNAs regulating tumor cell plasticity

\begin{tabular}{|c|c|c|}
\hline ncRNA & Summary & Reference \\
\hline \multicolumn{3}{|l|}{ miRNA } \\
\hline let-7 & $\begin{array}{l}\text { Down-regulated in breast cancer stem cells, regulate breast cancer stem cell differentiation by tar- } \\
\text { geting H-RAS and HMGA2 }\end{array}$ & [21] \\
\hline miR-17/miR-19b & Up-regulated in leukemia stem cells, regulate proliferation by targeting p21 & [23] \\
\hline $\operatorname{miR}-34$ & $\begin{array}{l}\text { Down-regulated in glioblastomas, regulate cell-cycle or apoptosis by targeting c-MET, Notch-1/2, } \\
\text { and CDK6 }\end{array}$ & {$[24]$} \\
\hline miR-181 & Up-regulated in hepatic cancer stem cells, maintain stemness by targeting CDX2, GATA6 and NLK & [25] \\
\hline miR-200 family & Down-regulated in EMT, regulate EMT by targeting ZEB1/2 & [26-28] \\
\hline miR-205 & Regulate ZEB1/2 and repress EMT & [26] \\
\hline $\operatorname{miR}-29 b$ & Reverse EMT and inhibit invasion in prostate cancer cells & [29] \\
\hline miR-1 & Target Slug to inhibit EMT in prostate adenocarcinoma cells & {$[31]$} \\
\hline $\operatorname{miR}-15 b$ & $\begin{array}{l}\text { Down-regulated in chemotherapy induced EMT, synergistically regulate chemoresistance and inva- } \\
\text { sion with miR-200b by targeting BMI1 }\end{array}$ & {$[32]$} \\
\hline miR-106b-25 & Target SMAD7 to induce EMT in TGF- $\beta$ treated breast cancer cells & [36] \\
\hline \multicolumn{3}{|l|}{ lncRNA } \\
\hline HOTAIR & $\begin{array}{l}\text { Up-regulated in metastatic breast tumors, indicate poor prognosis, enhance metastasis by modulat- } \\
\text { ing chromatin state }\end{array}$ & {$[40,41]$} \\
\hline MALAT1 & Up-regulated in metastatic lung adenocarcinomas, promote EMT by enhancing ZEB1/2 expression & {$[42,43]$} \\
\hline MEG3 & Involved in p53 signaling, suppress cell proliferation & {$[44-46]$} \\
\hline linc-p21 & Activated by p53 and mediate p53 induced apoptosis & [47] \\
\hline lncRNA-LET & Down-regulated by hypoxia and mediate hypoxia-induced invasion & [48] \\
\hline PRNCR1/PCGEM1 & Activate androgen-receptor pathway and facilitate castration-resistance in prostate cancer & [49] \\
\hline
\end{tabular}

codon in the translated sequences, this strategy is not applicable for long non-coding RNA transcripts as the transcripts themselves are the functional units. Other knock-out strategies such as disruption of core promoters of ncRNA genes are complicated by the nature that ncRNAs genes are often associated with protein-coding genes. Furthermore, silencing efficiencies of lncRNAs by siRNAs are often lower than proteins, because IncRNAs often form stable secondary structures that preclude binding of siRNAs to the target sequence. These challenges need to be overcome by developing new strategies to modulate ncRNA expression or building new models for functional studies in future.

This work was supported by the National Basic Research Program of China (2010CB912800, 2011CB504203), National Natural Science Foundation of China (81230060, 81261140373), National Science and Technology Major Special Project on New Drug Innovation of China (2011ZX09102010-02), Science Foundation of Guangdong Province (S2012030006287), and Key Laboratory of Malignant Tumor Gene Regulation and Target Therapy of Guangdong Higher Education Institutes, Sun Yat-sen University (KLB09001).

1 Scaffidi P, Misteli T. In vitro generation of human cells with cancer stem cell properties. Nat Cell Biol, 2011, 13: 1051-1061

2 Gupta P B, Chaffer C L, Weinberg R A. Cancer stem cells: Mirage or reality? Nat Med, 2009, 15: 1010-1012

3 Thiery J P, Acloque H, Huang R Y, et al. Epithelial-mesenchymal transitions in development and disease. Cell, 2009, 139: 871-890

4 Thiery J P, Sleeman J P. Complex networks orchestrate epithelial-mesenchymal transitions. Nat Rev Mol Cell Biol, 2006,7: 131-142

5 Peinado H, Olmeda D, Cano A. Snail, Zeb and bHLH factors in tumour progression: An alliance against the epithelial phenotype? Nat
Rev Cancer, 2007, 7: 415-428

6 Gibb E A, Brown C J, Lam W L. The functional role of long non-coding RNA in human carcinomas. Mol Cancer, 2011, 10: 38

7 Brosnan C A, Voinnet O. The long and the short of non-coding RNAs. Curr Opin Cell Biol, 2009, 21: 416-425

8 Okamura K, Chung W J, Ruby J G, et al. The Drosophila hairpin RNA pathway generates endogenous short interfering RNAs. Nature, 2008, 453: 803-806

9 Kawaji H, Hayashizaki Y. Exploration of small RNAs. PLoS Genet, 2008, 4: e22

10 Bartel D P. microRNAs: Target recognition and regulatory functions. Cell, 2009, 136: 215-233

11 Carthew R W, Sontheimer E J. Origins and mechanisms of miRNAs and siRNAs. Cell, 2009, 136: 642-655

12 Subramanyam D, Blelloch R. From microRNAs to targets: Pathway discovery in cell fate transitions. Curr Opin Genet Dev, 2011, 21: 498-503

13 Cheng J, Kapranov P, Drenkow J, et al. Transcriptional maps of 10 human chromosomes at 5-nucleotide resolution. Science, 2005, 308: 1149-1154

14 Wu Q, Kim Y C, Lu J, et al. Poly A- transcripts expressed in HeLa cells. PLoS ONE, 2008, 3: e2803

15 Guttman M, Amit I, Garber M, et al. Chromatin signature reveals over a thousand highly conserved large non-coding RNAs in mammals. Nature, 2009, 458: 223-227

16 Mattick J S. The genetic signatures of noncoding RNAs. PLoS Genet, 2009, 5: e1000459

17 Mattick J S, Makunin I V. Non-coding RNA. Hum Mol Genet, 2006, 15(Suppl 1): R17-R29

18 Qureshi I A, Mattick J S, Mehler M F. Long non-coding RNAs in nervous system function and disease. Brain Res, 2010, 1338: 20-35

19 Wilusz J E, Sunwoo H, Spector D L. Long noncoding RNAs: Functional surprises from the RNA world. Genes Dev, 2009, 23: 1494-1504

20 Shimono Y, Zabala M, Cho R W, et al. Downregulation of miRNA-200c links breast cancer stem cells with normal stem cells. Cell, 2009, 138: 592-603 
21 Yu F, Yao H, Zhu P, et al. let-7 regulates self-renewal and tumorigenicity of breast cancer cells. Cell, 2007, 131: 1109-1123

22 Yu F, Deng H, Yao H, et al. Mir-30 reduction maintains self-renewal and inhibits apoptosis in breast tumor-initiating cells. Oncogene, 2010, 29: 4194-204

23 Wong P, Iwasaki M, Somervaille T C P, et al. The miR-17-92 microRNA polycistron regulates MLL leukemia stem cell potential by modulating p21 expression. Cancer Res, 2010, 70: 3833-3842

24 Li Y, Guessous F, Zhang Y, et al. microRNA-34a inhibits glioblastoma growth by targeting multiple oncogenes. Cancer Res, 2009, 69: 7569-7576

25 Ji J, Yamashita T, Budhu A, et al. Identification of microRNA-181 by genome-wide screening as a critical player in EpCAM-positive hepatic cancer stem cells. Hepatology, 2009, 50: 472-480

26 Gregory P A, Bert A G, Paterson E L, et al. The miR-200 family and miR-205 regulate epithelial to mesenchymal transition by targeting ZEB1 and SIP1. Nat Cell Biol, 2008, 10: 593-601

27 Park S M, Gaur A B, Lengyel E, et al. The miR-200 family determines the epithelial phenotype of cancer cells by targeting the E-cadherin repressors ZEB1 and ZEB2. Genes Dev, 2008, 22: 894-907

28 Burk U, Schubert J, Wellner U, et al. A reciprocal repression between ZEB1 and members of the miR-200 family promotes EMT and invasion in cancer cells. EMBO Rep, 2008, 9: 582-589

29 Ru P, Steele R, Newhall P, et al. miRNA-29b suppresses prostate cancer metastasis by regulating epithelial-mesenchymal transition signaling. Mol Cancer Ther, 2012, 11: 1166-1173

30 Zhang J, Zhang H, Liu J, et al. miR-30 inhibits TGF- $\beta 1$-induced epithelial-to-mesenchymal transition in hepatocyte by targeting Snaill. Biochem Biophys Res Commun, 2012, 417: 1100-1105

31 Liu Y N, Yin J J, Abou-Kheir W, et al. miR-1 and miR-200 inhibit EMT via Slug-dependent and tumorigenesis via Slug-independent mechanisms. Oncogene, 2012, 32: 296-306

32 Sun L, Yao Y, Liu B, et al. miR-200b and miR-15b regulate chemotherapy-induced epithelial-mesenchymal transition in human tongue cancer cells by targeting BMI1. Oncogene, 2012, 31: 432-445

33 Mani S, Guo W, Liao M, et al. The epithelial-mesenchymal transition generates cells with properties of stem cells. Cell, 2008, 133: 704-715

34 Han M, Liu M, Wang Y, et al. Re-expression of miR-21 contributes to migration and invasion by inducing epithelial-mesenchymal transition consistent with cancer stem cell characteristics in MCF-7 cells. Mol Cell Biochem, 2012, 363: 427-436
35 Han M, Liu M, Wang Y, et al. Antagonism of miR-21 reverses epithelial-mesenchymal transition and cancer stem cell phenotype through AKT/ERK1/2 inactivation by targeting PTEN. PLoS ONE, 2012, 7: e39520

36 Smith A L, Iwanaga R, Drasin D J, et al. The miR-106b-25 cluster targets Smad7, activates TGF- $\beta$ signaling, and induces EMT and tumor initiating cell characteristics downstream of Six1 in human breast cancer. Oncogene, 2012, 31: 5162-5171

37 The ENCODE Project Consortium. An integrated encyclopedia of DNA elements in the human genome. Nature 2012, 489: 57-74

38 Ponting C P, Oliver P L, Reik W. Evolution and functions of long noncoding RNAs. Cell, 2009, 136: 629-641

39 Calin G A, Liu C G, Ferracin M, et al. Ultraconserved regions encoding ncRNAs are altered in human leukemias and carcinomas. Cancer Cell, 2007, 12: 215-229

40 Rinn J L, Kertesz M, Wang J K, et al. Functional demarcation of active and silent chromatin domains in human HOX loci by noncoding RNAs. Cell, 2007, 129: 1311-1323

41 Gupta R A, Shah N, Wang K C, et al. Long non-coding RNA HOTAIR reprograms chromatin state to promote cancer metastasis. Nature, 2010, 464: 1071-1076

42 Ji P, Diederichs S, Wang W, et al. MALAT-1, a novel noncoding RNA, and thymosin $\beta 4$ predict metastasis and survival in early-stage non-small cell lung cancer. Oncogene, 2003, 22: 8031-8041

43 Ying L, Chen Q, Wang Y, et al. Upregulated MALAT-1 contributes to bladder cancer cell migration by inducing epithelial-to mesenchymal transition. Mol BioSyst, 2012, 8: 2289-2294

44 Zhou Y, Zhong Y, Wang Y, et al. Activation of p53 by MEG3 non-coding RNA. J Biol Chem, 2007, 282: 24731-4742

45 Zhang X, Gejman R, Mahta A, et al. Maternally expressed gene 3, an imprinted noncoding RNA gene, is associated with meningioma pathogenesis and progression. Cancer Res, 2010, 70: 2350-2358

46 Braconi C, Kogure T, Valeri N, et al. microRNA-29 can regulate expression of the long non-coding RNA gene MEG3 in hepatocellular cancer. Oncogene, 2011, 30: 4750-4756

47 Huarte M, Guttman M, Feldser D, et al. A large intergenic noncoding RNA induced by p53 mediates global gene repression in the p53 response. Cell, 2010, 142: 409-419

48 Yang F, Huo X, Yuan S, et al. Repression of the long noncoding RNA-LET by histone deacetylase 3 contributes to hypoxia-mediated metastasis. Mol Cell, 2013, 49: 1083-1096

49 Yang L, Lin C, Jin C, et al. IncRNA-dependent mechanisms of androgen-receptor-regulated gene activation programs. Nature, 2013, 500: 598-602

Open Access This article is distributed under the terms of the Creative Commons Attribution License which permits any use, distribution, and reproduction in any medium, provided the original author(s) and source are credited. 